\title{
Checkpoint Inhibitors in Melanoma Patients with Underlying Autoimmune Disease
}

\author{
Hilary Dietz (D) \\ Sophia C Weinmann ${ }^{2}$ \\ April K Salamal \\ 'Division of Medical Oncology, Duke \\ University, Durham, NC, USA; ${ }^{2}$ Division \\ of Rheumatology and Immunology, Duke \\ University, Durham, NC, USA
}

Correspondence: April K Salama

Email april.salama@duke.edu

\begin{abstract}
The development of immune checkpoint inhibitors (ICI) has dramatically changed the clinical management of metastatic melanoma and other solid tumors. Despite exclusion from initial clinical trials, there is a growing body of retrospective data that suggest ICI can be used in patients with underlying autoimmune disease (AID) with a tolerable level of anticipated immune-related adverse events (irAEs) and a rate of severe irAEs comparable to that of patients without underlying AID. Coordination with other subspecialists and careful monitoring for irAEs is critical in safely managing these patients. Studies exploring novel approaches examining the use of targeted immunosuppressants in the prevention and management of irAEs, as well as multiple studies currently underway are aimed at establishing safe clinical practices when using ICI in patients with underlying AID.
\end{abstract}

Keywords: immune checkpoint inhibitor, melanoma, autoimmune disease

\section{Introduction}

In 2011, ipilimumab became the first approved treatment to demonstrate a survival benefit for advanced melanoma, marking the beginning of a new era in cancer immunotherapy. ${ }^{1}$ In the last decade, multiple immune checkpoint inhibitors (ICI) were developed and approved for clinical use across a number of cancer types. ${ }^{2}$ Ipilimumab blocks cytotoxic T-lymphocyte associated antigen 4 (CTLA-4), a molecule that functions as an immune checkpoint between T-cells and antigen presenting cells to dampen the immune response. ${ }^{1}$ Multiple drugs targeting the programmed cell death 1 (PD-1/PD-L1) pathway are also now routinely used in clinical practice, with pembrolizumab and nivolumab now approved for use in melanoma. ${ }^{3,4}$ Five-year survival data are now available from the original trials for advanced melanoma with these ICI, which show plateau of both progression-free and overall survival suggesting a population of patients who not only responded during treatment but have a durable reprogramming of their adaptive immune response after treatment with ICI. $^{5-7}$

Given the widespread indications for ICI in a variety of malignancies, up to $44 \%$ of the cancer population in the US may be candidates for therapy with a checkpoint inhibitor. ${ }^{8}$

Immune-related adverse events (irAEs), which result as collateral damage from immune system activation in patients receiving ICI, occur in most patients. Early studies in melanoma observed irAE-related toxicity in $60-70 \%$ of participants. ${ }^{9,10}$ These effects can range in severity from minor rash and loose stools, to severe irAEs requiring hospitalization with significant morbidity and mortality. ${ }^{10,11}$ 
There is significant concern regarding the use of checkpoint inhibitors in patients with underlying autoimmune disease (AID) that led to exclusion of these patients from many of the large, randomized trials used for initial FDA approval. ${ }^{12}$ This has then translated to a vacuum of information in clinical practice, due to limited reliable data documenting safety and efficacy of ICI in these patient populations. Additionally, there is limited information regarding the safety of ICI in these patients and best practices for toxicity monitoring and treatment. Moreover, there are no known biomarkers readily available for clinical practice that predict the incidence and severity of irAEs. Patients with known AID are often not considered candidates for potentially life-saving therapy with ICI and are often still excluded from clinical trials despite continued, albeit cautious use in clinical practice. ${ }^{13}$

In this review, we will examine the available data describing outcomes of patients with underlying AIDs treated with ICI and address the need for standardized guidelines for selection and management of these patients. Although these patients were universally excluded from initial trials, retrospective review of management outcomes shows there is a population of individuals with AIDs who can tolerate and benefit from therapy with ICI.

\section{Pathophysiology of the Development of irAEs}

Development of autoimmune manifestations during treatment with ICI therapy is multifactorial and hypotheses include the increase in pro-inflammatory cytokines due to T-cell activation and direct T-cell effects against host tissue. ${ }^{9}$ There is a growing body of evidence with multiple studies in the pre-clinical setting implicating CTLA-4 and PD-1 pathways in the development of immunedysregulated phenotypes in animal models and humans. ${ }^{14-16}$ CTLA-4 knockout mice develop uniformly fatal lymphoproliferation with multiorgan dysfunction, ${ }^{14}$ whereas PD-1 knockout mice can develop autoimmune diseases such as dilated cardiomyopathy, glomerulonephritis and autoimmune diabetes, suggesting the distinct, but critical roles these loci play in regulating immune processes that could lead to pathologic states. ${ }^{15,16}$ Single nucleotide polymorphisms (SNPs) at these loci have also been implicated in autoimmune disease in humans. ${ }^{17,18}$ Other SNPs are implicated in increased risk for breast, gastrointestinal and head and neck cancers, highlighting the role of disrupted immune surveillance in the development of malignancy. ${ }^{19,20}$

Development of certain irAEs mimicking known AID correlates with response to immunotherapy in patients with melanoma. There is a well-documented relationship between checkpoint-inhibitor induced cutaneous toxicity, specifically vitiligo, and improved tumor response. ${ }^{21-23}$ A retrospective study of 346 patients with melanoma showed that those who developed gastrointestinal immune adverse events had a statistically significant improvement in progression-free and overall survival compared to those who did not. ${ }^{24}$ Moreover, the severity of the irAE appeared to correlate with benefit: patients who developed higher-grade diarrhea had a statistically significant improvement in overall survival. ${ }^{24}$ Multiple prospective studies however have demonstrated no difference in progression-free and overall survival in patients with advanced melanoma who stopped treatment due to toxicity versus those who did not. ${ }^{6,25}$ Conversely, secondary analysis of keynote-054 comparing relapse-free survival in patients who received adjuvant pembrolizumab after surgical resection of stage III melanoma showed longer relapse-free survival in patients who developed irAEs on ICI. ${ }^{26}$ This implies that in a carefully monitored clinical setting, some level of immune mediated toxicity can be safely tolerated in order to achieve adequate treatment response, and with more rigorous data, this could have implications for patients with pre-existing AID as well.

\section{Immunotherapy in Patients with Underlying Autoimmune Disease}

Clinical trials have largely excluded patients with preexisting AID due to concerns for increased toxicity; however, there is growing evidence that ICI can be used in patients with underlying AID with careful consideration and planning. While some patients with mild AID not requiring systemic immunosuppressive therapy are allowed in trials, there are many patients who are ultimately deprived of the opportunity. The main underlying concerns are the increased risk and severity of irAEs, the risk of flares of underlying AID, and the possibility that immunosuppressive treatment for AID will decrease the efficacy of ICI, leading to worse outcomes. While there are alternatives to consider in some situations, treatment of melanoma is still heavily reliant on ICI for disease control particularly in the metastatic setting with increasing evidence supporting ICI in adjuvant settings. ${ }^{27-29}$ 
Multiple studies attempted to characterize the risk of AID flare and de novo irAEs in these patients, relying on retrospective review of pre-existing clinical data. Studies discussed in this review are summarized in Table 1. Kehl et al performed a large retrospective case series from a private insurance database, in which 4438 patients were identified who had received ICI for malignancy between June 2011 and June 2017. There were 283 patients (6\%) identified with AID based on ICD 9/ICD 10 codes. These patients had a statistically significant increase in hospitalization for irAEs and need for corticosteroid treatment compared to those with no identified AID. ${ }^{30}$ Another study by Bender et al examined claims data and electronic health records to compare patients with and without AID undergoing therapy with ICI for melanoma. Among patients receiving anti-PD-1 therapy, which generally has a more favorable toxicity profile than anti-CTLA-4 or dual checkpoint inhibitor therapy, patients with underlying AID once again had a statistically significant increase in hospitalization and treatment with oral prednisone compared to patients without underlying AID. ${ }^{31,32}$ These studies were able to capture a significant population of patients and compare basic metrics such as hospitalization rates with patients not affected by AID, but were limited by the use of insurance and pharmacy databanks as the source of data, relying on the use of specific diagnostic codes to determine inclusion of a patient in a specific group. Additionally, there is no opportunity for review of individual cases or specific clinical data such as type and severity of irAE that would prove valuable in making clinical decisions and advising patients on their risk.

Further studies have included case reviews to describe clinical outcomes in these patients. Cortellini et al examined 751 patients in Italy, $85(11.3 \%)$ with preexisting AID, who received anti PD-1 immunotherapy for advanced cancer. They found patients in their study had no statistically significant increase in grade 3 or 4 toxicities compared to individuals without autoimmune disease. Furthermore, there was a statistically significant difference in composite irAE of any type and grade 1 or 2 toxicities compared to non-affected individuals. ${ }^{33}$ This group did not distinguish between flare of underlying disease and de novo irAE in their statistical analysis, but all grade 3 or 4 events in patients with AID were flares of their underlying diseases. While this study showed patients with preexisting AID did have an increase in low grade irAEs, AID was not an independent factor in progression-free survival or overall survival. ${ }^{33}$
The most comprehensive examination of ICI and AID in patients with advanced cancer was a metaanalysis performed by Abdel-Wahab et al. ${ }^{34}$ They evaluated data from 49 publications documenting 123 cases of patients with AID receiving ICI, and $83.7 \%$ of cases represented patients with melanoma. The most common pre-existing conditions represented in this analysis were psoriatic arthritis and psoriasis (22.8\%), rheumatoid arthritis $(16.3 \%)$, autoimmune thyroid disease $(8.9 \%)$, inflammatory bowel disease (10.6\%), and multiple sclerosis $(4.9 \%)$. Treatment consisted of ipilimumab in $44.7 \%$, anti-PD- 1 therapy in $52 \%$, and anti-PD-L1 in $19.5 \%$ of patients. Only $2.4 \%$ of patients received dual checkpoint inhibitor therapy with ipilimumab and nivolumab. The majority of patients $(84 \%)$ had been treated for their underlying AID at some point, and $43 \%$ of patients were still on active therapy at the time of initiating immunotherapy. The majority of patients (75\%) developed an adverse event with 50 (41\%) experiencing flare of their AID and 31 (25\%) developing a de novo irAE, while eleven (9\%) patients had both a flare and a de novo event. There was no difference in irAEs observed between patients with active vs inactive AID ( $67 \%$ vs $75 \%)$, and there was a trend toward fewer adverse events if patients were on therapy for their AID at the initiation of ICI $(59 \%$ vs $83 \%)$. In contrast, Leonardi et al demonstrated that patients with well controlled pre-existing AID at the time of initiation of ICI therapy for non-small cell lung cancer had a statistically significant decreased incidence of flare of pre-existing disease, with a $50 \%$ incidence in symptomatic patients versus only an $18 \%$ incidence in asymptomatic patients. $^{35}$

These studies suggest that across all cancer types there are patients with AID who can tolerate ICI therapy with rates of severe irAE like that of the general population. Patients with AID have an increased risk of irAEs with a majority representing grade 1-2 toxicity that can be managed with steroids in an office setting. ${ }^{30,32}$ Abdel-Wahab et al and Leonardi et al were able to provide insight into the relationship between disease control at the time of treatment and irAEs, while Cortellini et al provided evidence that there is no harmful effect on overall survival in patients with underlying AID when examined across cancer types. ${ }^{33-35}$ While these retrospective studies are informative, prospective studies are needed in this patient population for clarification of conflicting data regarding 
Table I Summary of Case Series Cited in This Review

\begin{tabular}{|c|c|c|c|c|c|c|c|}
\hline Study & Patients, $\mathrm{n}$ & Tumors (\%) & ICI Used (\%) & AID (\%) & $\begin{array}{l}\text { Baseline AID } \\
\text { Treatment (\%) }\end{array}$ & $\begin{array}{l}\text { AID } \\
\text { Flare (\%) }\end{array}$ & irAEs (\%) \\
\hline $\begin{array}{l}\text { Cortellini } \\
\text { et } \mathrm{al}^{33}\end{array}$ & 85 & $\begin{array}{l}\operatorname{NSCLC}(65.5), \\
\text { melanoma (21.2), } \\
\operatorname{RCC}(12.5)\end{array}$ & $\begin{array}{l}\text { Pembrolizumab } \\
\text { (24.2), nivolumab } \\
\text { (75.8) }\end{array}$ & $\begin{array}{l}\text { AIT (60), psoriasis (13), } \\
\text { rheumatologic (II.8), } \\
\text { IBD (3.5) }\end{array}$ & $\begin{array}{l}\text { Any (17.6) } \\
\text { Steroids (13) } \\
\text { Other } \\
\text { immunosuppressant (3.5) }\end{array}$ & 47 & 65.9 \\
\hline $\begin{array}{l}\text { Abdel- } \\
\text { Wahab } \\
\text { et } \mathrm{al}^{34}\end{array}$ & 123 & $\begin{array}{l}\text { Melanoma (83.7), } \\
\text { lung (13), RCC } \\
(2.4) \text { merkel cell } \\
(0.8)\end{array}$ & $\begin{array}{l}\text { Ipilimumab (44.7) } \\
\text { Nivolumab (27) } \\
\text { Pembrolizumab (19) } \\
\text { Atezolizumab (0.8) } \\
\text { Combination (2.4) }\end{array}$ & $\begin{array}{l}\text { Psoriasis and psoriatic } \\
\text { arthritis (22.8) } \\
\text { RA (16.3), AIT (8.9), } \\
\text { IBD (I0.6), MS (4.9), } \\
\text { Sarcoidosis (4.I), MG } \\
(3.3)\end{array}$ & $\begin{array}{l}\text { Any (26.7), Steroids } \\
(20.8), \text { Synthetic } \\
\text { DMARDs (14), Biologic } \\
\text { DMARDs (1.0), Other } \\
(4.0)\end{array}$ & 49.5 & 34 \\
\hline $\begin{array}{l}\text { Leonardi } \\
\text { et } \mathrm{al}^{35}\end{array}$ & 56 & NSCLC (100) & PD-I/PD-LI (100) & $\begin{array}{l}\text { Rheumatologic (45), } \\
\text { dermatologic (29), AIT } \\
\text { (16), IBD (II), } \\
\text { neurologic (5) }\end{array}$ & $\begin{array}{l}\text { Any (20), Steroids (7), } \\
\text { Steroid sparing agents } \\
(12.5)\end{array}$ & 23 & 38 \\
\hline $\begin{array}{l}\text { Johnson } \\
\text { et } \mathrm{al}^{36}\end{array}$ & 30 & Melanoma $(100)$ & Ipilimumab (100) & $\begin{array}{l}\text { RA (20), psoriasis (I7), } \\
\text { IBD (20), MS (7), AIT } \\
\text { (I0), sarcoidosis (7), } \\
\text { SLE (7), other (23) }\end{array}$ & $\begin{array}{l}\text { Any (73), Steroids (33), } \\
\text { DMARDs (43) }\end{array}$ & 27 & 33 \\
\hline $\begin{array}{l}\text { Kahler } \\
\text { et al }\end{array}$ & 41 & Melanoma (100) & Ipilimumab (100) & $\begin{array}{l}\text { RA (15), psoriasis (I7), } \\
\text { IBD (7), AIT(37), } \\
\text { sarcoidosis (5), MS (2.5) }\end{array}$ & $\begin{array}{l}\text { Any (27), Steroids (14.5), } \\
\text { 5-ASA agents (7), } \\
\text { Methotrexate ( } 2.4)\end{array}$ & 29.2 & 29.2 \\
\hline $\begin{array}{l}\text { Menzies } \\
\text { et } \mathrm{al}^{38}\end{array}$ & 52 & Melanoma $(100)$ & Anti-PD-I & $\begin{array}{l}\text { RA ( } 25) \text {, IBD }(9.6) \text {, } \\
\text { psoriasis (II.5), } \\
\text { sarcoidosis (5.8), other } \\
\text { rheumatologic (2I), } \\
\text { neurologic (10), AIT (8) }\end{array}$ & $\begin{array}{l}\text { Any (38), Steroids (17), } \\
\text { Steroid sparing (10), } \\
\text { Steroids +SSA (10) }\end{array}$ & 38 & 29 \\
\hline $\begin{array}{l}\text { Grover } \\
\text { et al }\end{array}$ & 25 & $\begin{array}{l}\text { Melanoma (40), } \\
\text { lung (28), CRC (8), } \\
\text { GU (8), gyn } \\
\text { (4), merkel cell (4) }\end{array}$ & $\begin{array}{l}\text { Ipilimumab (4) } \\
\text { Anti PD-I/PD-LI } \\
(88) \\
\text { Combination (8) }\end{array}$ & $\begin{array}{l}\text { IBD (84), microscopic } \\
\text { colitis (16) }\end{array}$ & $\begin{array}{l}\text { Any (40), 5-ASA agents } \\
\text { (28), Steroids (8), Anti } \\
\text { TNF (8) }\end{array}$ & 28 & No data \\
\hline $\begin{array}{l}\text { Abu-Sbeih } \\
\text { et } \mathrm{al}^{4 l}\end{array}$ & 102 & $\begin{array}{l}\text { Melanoma (44), } \\
\text { NSCLC (23), GI } \\
\text { (17), GU (7), H+N } \\
\text { (4), other (6) }\end{array}$ & $\begin{array}{l}\text { CTLA-4 (7) } \\
\text { PD-I/PD-LI (83) } \\
\text { Combination (10) }\end{array}$ & IBD (100) & $\begin{array}{l}\text { Any (58), mesalamine } \\
\text { (36), other } \\
\text { immunosuppression (22) }\end{array}$ & 41 & 31 \\
\hline $\begin{array}{l}\text { Efuni } \\
\text { et } \mathrm{al}^{50}\end{array}$ & 22 & $\begin{array}{l}\text { Melanoma (32), } \\
\text { NSCLC (32), } \\
\text { merkel cell (9), H } \\
+\mathrm{N}(9), \mathrm{GU}(10), \\
\text { gyn (5) }\end{array}$ & $\begin{array}{l}\text { Ipilimumab (23) } \\
\text { Nivolumab (4I) } \\
\text { Pembrolizumab (59) }\end{array}$ & $\begin{array}{l}\text { RA }(100) \text {, other AID } \\
\text { present as well ( } 32)\end{array}$ & $\begin{array}{l}\text { Any (73), Steroids (55), } \\
\text { Hydroxychloroquine } \\
\text { (14), Methotrexate (32), } \\
\text { Other (15) }\end{array}$ & 55 & 32 \\
\hline
\end{tabular}

Abbreviations: NSCLC, non-small cell lung cancer; RCC, renal cell carcinoma; GU, genitourinary; CRC, colorectal cancer; gyn, gynecologic; AIT, autoimmune thyroid disease; RA, rheumatoid arthritis; MS, multiple sclerosis; IBD, inflammatory bowel disease; DMARD, disease-modifying antirheumatic drug.

the use of ICI therapy in patients with pre-existing AID. Further studies are necessary to effectively describe the relative risk of irAEs and efficacy of ICI treatment in patients with active AID not on therapy, AID controlled on therapy, and AID off therapy at time of initiation of ICI.

\section{Immunotherapy in Patients with Melanoma and Underlying Autoimmune Disease}

Given the lack of alternative treatment options and broad use in clinical practice, there have been several 
retrospective case studies reviewing ICI and AID in patients with malignant melanoma. While there are alternatives to consider in some situations, treatment of melanoma is still heavily reliant on ICI for disease control particularly in the metastatic setting with increasing evidence supporting use in adjuvant settings. ${ }^{27-29}$

Johnson et al performed a retrospective review of cases of nine academic medical centers in patients with melanoma who received ipilimumab despite underlying AID. ${ }^{36}$ The most common AIDs in the 30 patients included were rheumatoid arthritis in 6 patients, psoriasis in 5, and inflammatory bowel disease in 6. Eight patients (27\%) developed a flare of their AID, and 10 patients (33\%) suffered a grade 3 or 4 immune related adverse event, which was either a de novo irAE or a flare of their preexisting AID. Importantly, 15 patients (50\%) had no immune-related complications. There was one death in this series in a patient with psoriasis who developed grade 5 colitis, after a diagnostic delay. Kahler et al examined data from 14 skin cancer centers in Germany in patients with AID who received ipilimumab. ${ }^{37}$ Forty-one patients were identified, but a considerable proportion (41\%) had autoimmune thyroiditis as their main AID, and $27 \%$ had rheumatoid arthritis. Approximately $30 \%$ of patients developed a flare of their AID and another 30\% had a de novo irAE. Nearly half of patients had no immune complications, a similar proportion to the previously described cohort. ${ }^{36,37}$ Overall, these studies reinforce similar findings that roughly $50 \%$ of patients with AID and melanoma started on ipilimumab will have an irAE, either de-novo or an AID flare.

An international retrospective cohort of melanoma patients with pre-existing AID receiving pembrolizumab or nivolumab was examined by Menzies et al. In this study, 52 patients across 13 academic referral centers were identified after receiving anti-PD-1 therapy. Only $38 \%$ of patients had a flare of their underlying disease while $29 \%$ developed de novo irAEs. ${ }^{38}$ Notably, there was an increase in irAEs in patients on therapy for AID at the time they initiated ICI, which is the opposite from the observation made by Abdel-Wahab et al, suggesting the risk of developing an irAE is complex and may be influenced by both the overall severity of underlying AID and disease control at the start of ICI therapy. ${ }^{34,38}$

These studies suggest that while there are patients with underlying AIDs who develop worsening symptoms while on ICI, there may not be a clinically significant difference in the rate of severe de novo irAEs. The increase in toxicity was largely represented by patients who experienced grade 1-2 irAEs. However, these studies are limited in their ability to directly guide clinical decision making. While these studies include data from multiple centers, the number of cases examined remains limited. Similarly, the retrospective design allows for significant bias and confounding variables. The data are difficult to generalize to individual patients given the heterogeneity of the reported underlying autoimmune disorders, characteristics of their malignancy, and clinical status. While these studies included patients with more prevalent autoimmune diseases; namely rheumatoid arthritis, IBD and MS, there were also patients included with thyroiditis, severe asthma and hematological autoimmune disorders such as immune thrombocytopenic purpura and cold agglutinin disease. The veritable mix of patients included significantly diminishes one's ability to generalize the results to their specific patient populations, and more studies are needed in patients with specific autoimmune diseases in order to best guide clinical decisions.

\section{ICI in Inflammatory Bowel Disease}

Colitis is a common irAE in patients receiving ICI therapy that causes significant morbidity and potential mortality, making patients with pre-existing ulcerative colitis (UC) and Crohn's disease a therapeutic challenge for oncologists. In one case report, a patient developed grade 3 colitis after treatment with ipilimumab for melanoma in the setting of active UC. The patient was treated with TNF- $\alpha$ blockade and colectomy before successfully tolerating rechallenge with anti-CTLA-4 therapy resulting in complete response $(\mathrm{CR})$ of the melanoma. ${ }^{39} \mathrm{~A}$ meta-analysis of multiple case series showed 8 of $13(62 \%)$ patients with inflammatory bowel disease (IBD) developed an adverse event, including 5 (39\%) who had an exacerbation of their underlying colitis with one of these cases resulting in a life-threatening bowel perforation. ${ }^{28}$ Another retrospective review included 21 patients with IBD receiving ICI in which only 4 patients (19\%) developed a flare of their underlying colitis. ${ }^{40}$ Of note, the 2 patients in this series receiving dual checkpoint with ipilimumab and nivolumab developed a flare of their colitis, consistent with the increased toxicity seen in the general population with combined checkpoint blockade. ${ }^{31}$ A large, multi-center, retrospective review from Abu-Sbeih et al identified 102 patients from fourteen treatment centers with confirmed IBD receiving either anti-CTLA-4 or anti-PD-1/PD-L1 therapy. $^{41} 45 \%$ of patients in this cohort had melanoma; 
and a majority (83\%) of patients were on single agent PD1/PD-L1 therapy for their malignancy. Only $58 \%$ were on active treatment for their IBD at the time ICI was initiated. This study included statistical comparison to patients receiving ICI without underlying IBD and showed a statistically significant difference in rate of GI adverse events between these groups ( $41 \%$ vs $11 \%$ ). Univariate analysis showed CTLA-4 blockade had higher odds of GI adverse events. Otherwise, there was no statistically significant association between odds of GI adverse events and several other important clinical metrics including whether patients were on therapy for IBD before ICI was initiated, pretreatment endoscopic findings, recent active IBD, colonic involvement, extraintestinal involvement of IBD or known anatomical complications of IBD (ie, stricture or fistula). The authors do note that although there were limited data, 5 of the 7 patients who had severe features on endoscopic examination before initiating treatment with ICI developed GI adverse effects, and further studies investigating the predictive role of pretreatment endoscopy could provide useful insight. ${ }^{41}$

Two case reports have documented a strategy to prevent colitis flares through the simultaneous administration of an ICI plus a biologic, either tocilizumab or vedolizumab in patients with underlying Crohn's disease. ${ }^{42,43}$ These cases both resulted in durable tumor response with only a mild flare of the underlying colitis. This highlights the complexity regarding the overlap in the mechanism of action of ICI, with a desire to generate anti-tumor immune responses, against the pathophysiology of IBD, but may also offer practical alternative strategies to manage the potential toxicity of ICI therapy in patients with IBD. While ICI therapy in patients with underlying IBD can be considered safe and effective in some patients, there is a risk for clinically significant colitis flares as well as development of de-novo irAEs in these patients. Coordination with an IBD specialist and careful monitoring for diarrhea and extra-intestinal symptoms should be included as part of a multi-disciplinary approach in the care of these patients.

\section{ICI in Rheumatoid Arthritis}

Rheumatoid arthritis (RA) is a rare autoimmune disease, and studies have validated the increased malignancy risk in these patients, specifically an increased risk of nonHodgkin's lymphoma, non-melanoma skin cancer, and lung cancer. ${ }^{44-46}$ ICI-associated inflammatory arthritis is well described, and symptoms can persist for months to years after cessation of immunotherapy. ${ }^{47}$ Moreover, patients with pre-existing RA may experience disease flares on ICI therapy. ${ }^{4-49}$ The meta-analysis by AbdelWahab et al included 20 patients with RA, 15 of whom developed irAEs, either disease flare or de novo irAE with 12 requiring steroids and 2 requiring infliximab for refractory colitis. ${ }^{34}$ Multiple retrospective studies described previously noted an increased rate of disease flare in rheumatic diseases compared to dermatologic, neurologic or gastrointestinal autoimmune diseases in the setting of ICI therapy. ${ }^{37,38}$

One retrospective cohort study included patients with RA undergoing therapy with ICI for solid malignancy, and among 22 patients, 7 received therapy for melanoma. ${ }^{50}$ Seventy-three percent of patients experienced either disease flare or a de novo irAE, which is a higher incidence than some larger cohort studies including one heterogenous population of patients based on underlying AID. ${ }^{36,50}$ Only two patients developed grade 3 toxicity with colitis and dermatitis representing the most common adverse events. Twelve (55\%) patients experienced an RA flare, and 10 of these patients $(83 \%)$ required oral steroids. While the majority of complications were treated with a short course of steroids and resolved, 5 patients required cessation of ICI therapy due to disease flare or de novo irAE, but only 1 required permanent discontinuation. ${ }^{50}$

Another smaller series from Lee et al described a cohort of eight patients with rheumatoid arthritis undergoing therapy with ipilimumab for melanoma. ${ }^{51}$ In this series, only 2 patients received the planned four cycles of ipilimumab with five discontinuing early due to intolerable side effects, two with grade 3 arthritis and four with grade 3 colitis. Additionally, adverse events were common, with half of the patients developing more than one irAE.

The clinical significance of the reported differences in these case series remains unclear. Overall, these studies highlight the need for studies with larger sample sizes in order to adequately characterize and describe the risk of irAEs and disease flares in patients with rheumatoid arthritis and other rheumatic diseases. In these small series, there is an increased incidence of irAEs in patients with RA compared to those with other underlying AIDs. Despite these irAEs, it is likely that some patients with RA could tolerate and benefit from therapy with ICI; however, further investigation is necessary to determine how to monitor and manage complications in these patients as part of a collaborative effort between medical oncology and rheumatology. 


\section{Multiple Sclerosis and Other Neuroinflammatory Disorders}

Data regarding the management of melanoma patients with underlying multiple sclerosis (MS), myasthenia gravis (MG), and other neuro-inflammatory disorders are limited, with no clear consensus on how to select patients for ICI therapy. Information is limited to observational data, case reports, and patients who are included in cohort studies having significant variation in clinical presentation and outcome. These disorders should be of particular concern for medical oncologists given the risk of morbidity and even mortality with exacerbation of a pre-existing neurological autoimmune disease.

There are multiple accounts of MS patients undergoing therapy with no complication or remaining on therapy with interferon beta during ICI therapy, but also reports of rapid disease progression on ICI. ${ }^{52}$ Garcia et al performed an institutional and FAERS review of patients with adverse reaction to ICI and a history of multiple sclerosis. In total 14 cases were identified with seven occurring in patients receiving therapy for melanoma. Adverse events included altered mental status, increased weakness and sensory disturbance attributed to ICI-induced flare of their underlying MS. Three patients had progressive MS (23\%), and two patients died as a result (15\%). ${ }^{53}$

Outside of MS and MG there are cases of ICI used in other neurological autoimmune disorders, such as neuromyelitis optica and transverse myelitis, but no large cohorts of data. ${ }^{37}$ Case reports of disorders such as MG show the significant risk ICI therapy poses to these populations. Multiple case reports describe patients with metastatic melanoma who had significant worsening of underlying MG with pembrolizumab monotherapy resulting in hospital admission, respiratory failure requiring ICU admission and non-invasive positive pressure ventilation, as well as therapy with IVIG. ${ }^{54,55}$ In Abdel-Wahab et al's study, four patients with myasthenia gravis were included and all developed immune toxicity with three developing flares of myasthenia gravis and one with new granulomatous infiltration of the pleura attributed to irAE. ${ }^{34}$

The neurological autoimmune disorders represent an area of clinical practice where there is little consensus regarding safety of ICI among available retrospective data. Therapy with an ICI in these patients could be considered, but active involvement from the patient's neurologist as well as a proactive plan for symptom monitoring and control should be considered before initiation of therapy.

\section{Future Directions}

There are currently two separate single arm, prospective, phase $1 \mathrm{~b}$ clinical trials evaluating the use of nivolumab in patients with dermatomyositis, systemic sclerosis, rheumatoid arthritis, systemic lupus erythematous, multiple sclerosis, Sjogren's syndrome and inflammatory bowel disease who have a malignancy known to be sensitive to anti-PD -1 therapy (NCT03816345, NCT03656627). These studies will provide meaningful data regarding frequency of adverse events both from disease flare and de novo irAEs gathered in the controlled setting of a clinical trial, a critical first step in answering the many questions that remain.

While there is no consensus or randomized data to support strategies to prevent and treat irAEs and disease flares in patients with autoimmune diseases on ICI, multiple strategies have been proposed including B cell directed therapies such as rituximab for patients with SLE, the gut specific anti- $\alpha 4 \beta 7$ integrin vedolizumab in patients with IBD and anti-IL-6 in rheumatoid arthritis. ${ }^{56}$ These proposals are based mainly on preclinical data and anecdotal evidence from individual cases, but this approach does take advantage of the sheer complexity of the adaptive immune system, potentially enabling the generation of a tumor-specific immune response, while minimizing irAEs via alternative pathways. In the future, it is possible that selective immunomodulators will be used concurrently with ICI in these patients to prevent or treat irAEs. Furthermore, while there are currently no FDA-approved assays to predict irAEs in patients with or without underlying AID, this is an ongoing area of research with multiple cytokines found to correlate with increased risk of irAEs. ${ }^{57}$ This may allow medical oncologists to be proactive in the prevention of irAEs rather than reactive after their development.

\section{Conclusion}

Currently, there are limited data to guide clinical decisionmaking regarding selection of patients with underlying AID for cancer treatment with checkpoint inhibition; however, it appears that there is a population of these patients who can tolerate ICI therapy with minimal to no irAEs. Existing data are in the form of retrospective case series and meta-analyses with likely selection bias and heterogenous reports of severity of autoimmune disease and malignancy. It is impossible to truly determine the frequency of autoimmune flares and de novo irAEs outside the setting of a prospective cohort study, which will also be essential for development of standardized clinical protocols to monitor these patients while initiating therapy. 
It is possible to give checkpoint inhibitors safely to patients with underlying autoimmune diseases; however, decisions must be individualized as limited concrete data exist to guide patient selection. It is also possible that novel strategies to modulate checkpoint blockade toxicity may diminish the risk of irAEs, both pre-existing disease flare and de novo irAEs, in this patient population in the future.

Considerations for initiating immune therapy in patients with underlying AID should include an emphasis on transparency and shared decision making between the patient and provider, and if possible, the patient should have well-controlled AID before starting treatment with ICI. Coordinated care between oncology and other subspecialists as appropriate, is critical for toxicity monitoring and early intervention in order to maximize clinical benefit while mitigating risk.

\section{Disclosure}

Dr April K Salama reports grants from Bristol Myers Squibb, grants from Immunocore, grants from Merck, grants from Nektar Therapeutics, personal fees from Novartis, personal fees from Pfizer, personal fees from Iovance, personal fees from Regeneron, outside the submitted work. The authors report no other conflicts of interest in this work.

\section{References}

1. Hodi FS, O'Day SJ, McDermott DF, et al. Improved survival with ipilimumab in patients with metastatic melanoma. $N$ Engl $\mathrm{J} \mathrm{Med.}$ 2010;363(8):711-723. doi:10.1056/NEJMoa1003466

2. Vaddepally RK, Kharel P, Pandey R, Garje R, Chandra AB. Review of indications of FDA-approved immune checkpoint inhibitors per NCCN guidelines with the level of evidence. Cancers. 2020;12 (3):738. doi:10.3390/cancers 12030738

3. Wolchok JD, Chiarion-Sileni V, Gonzalez R, et al. Overall survival with combined nivolumab and ipilimumab in advanced melanoma. $N$ Engl J Med. 2017;377(14):1345-1356. doi:10.1056/NEJMoa170 9684

4. Ribas A, Puzanov I, Dummer R, et al. Pembrolizumab versus investigator-choice chemotherapy for ipilimumab-refractory melanoma (KEYNOTE-002): a randomized, controlled, Phase 2 trial. Lancet Oncol. 2015;16(8):908-918. doi:10.1016/S1470-2045(15) 00083-2

5. Hamid O, Robert C, Daud A, et al. Five-year survival outcomes for patients with advanced melanoma treated with pembrolizumab in KEYNOTE-001. Ann Oncol. 2019;30:582-588. doi:10.1093/annonc/ mdz011

6. Larkin J, Chiarion-Sileni V, Gonzalez R, et al. Five-year survival with combined nivolumab and ipilimumab in advanced melanoma. $N$ Engl $J$ Med. 2019;318(16):1535-1546. doi:10.1056/NEJMoa1910836

7. Wolchok JD, Weber JS, Maio M, et al. Four-year survival rates for patients with metastatic melanoma who received ipilimumab in Phase II clinical trials. Ann Oncol. 2013;24(8):2174-2180. doi:10.1093/ annonc/mdt161
8. Haslam A, Prasad V. Estimation of the percentage of US patients with cancer who are eligible for and respond to checkpoint inhibitor immunotherapy drugs. JAMA Net Open. 2019;2(5):e192535. doi:10.1001/jamanetworkopen.2019.2535

9. Postow MA, Sidlow R, Hellmann MD. Immune-related adverse events associated with immune checkpoint blockade. $N$ Engl J Med. 2018;378(2):158- 168. doi:10.1056/NEJMra1703481

10. Wang Y, Zhou S, Yang F, et al. Treatment-related adverse events of PD-1 and PD-L1 inhibitors in clinical trials: a systematic review and meta-analysis. JAMA Oncol. 2019;5(7):1008-1019.

11. Wang DY, Salem JE, Cohen JV, et al. Fatal toxic effects associated with immune checkpoint inhibitors: a systematic review and meta-analysis. JAMA Oncol. 2018;4(12):1721-1728. doi:10.1001/jamaoncol.2018.3923

12. Kennedy LC, Bhatia S, Thompson JA, Grivas P. Preexisting autoimmune disease: implications for immune checkpoint inhibitor therapy in solid tumors. J Natl Compr Cancer Net. 2019;17(6):750-757. doi:10.6004/jncen.2019.7310

13. Pantuck M, McDermott D, Drakaki A. To treat or not to treat: patient exclusion in immune oncology clinical trials due to preexisting autoimmune disease. Cancer. 2019;125:3506-3513. doi:10.1002/cncr.32326

14. Tivol EA, Borriello F, Schweitzer AN, Lynch WP, Bluestone JA, Sharpe AH. Loss of CTLA-4 leads to massive lymphoproliferation and fatal multiorgan tissue destruction, revealing a critical negative regulatory role of CTLA-4. Immunity. 1995;3(5):541-547. doi:10.1016/1074-7613(95)90125-6

15. Nishimura $H$, Nose $M$, Minato N, Honjo T. Development of lupus-like autoimmune diseases by disruption of the PD-1 gene encoding an ITIM motif-carrying immunoreceptor. Immunity. 1999;11(2):141-151. doi:10.1016/S1074-7613(00)80089-8

16. Nishimura H, Okazaki T, Tanaka Y, et al. Autoimmune dilated cardiomyopathy in PD-1 receptor-deficient mice. Science. 2001;291:319-322. doi:10.1126/science.291.5502.319

17. Ueda H, Howson JMM, Esposito L, et al. Association of the T-cell regulatory gene CTLA4 with susceptibility to autoimmune disease. Nature. 2003;423(6939):506-511. doi:10.1038/nature01621

18. Zamani MR, Aslani S, Salmaninejad A, Javan MR, Reaei N. PD-1/ PD-L and autoimmunity: a growing relationship. Cell Immunol. 2016;310:27-41. doi:10.1016/j.cellimm.2016.09.009

19. Fang M, Huang W, Mo D, Zhao W, Huang R. Association of five Snps in cytotoxic T-lymphocyte antigen 4 and cancer susceptibility: evidence from 67 studies. Cell Physiol Biochem. 2918;47 (1):414-427. doi:10.1159/000489953

20. Li J, Wang W, Sun Y, Zhu Y. CTLA-4 polymorphisms and predisposition to digestive system malignancies: a meta-analysis of 31 published studies. World J Surg Oncol. 2020;18(1):55. doi:10.1186/ s12957-020-1806-2

21. Nakano E, Takahashi A, Namikawak K, et al. Correlation between cutaneous adverse events and prognosis in patients with melanoma treated with nivolumab: a single institutional retrospective study. J Dermtol. 2020;47(6):622-628. doi:10.1111/1346-8138.15309

22. Hua C, Boussemart L, Mateus C, et al. Association of vitiligo with tumor response in patients with metastatic melanoma treated with pembrolizumab. JAMA Dermatol. 2016;152(1):45-51. doi:10.1001/ jamadermatol.2015.2707

23. Teulings HE, Limpens J, Jansen S, et al. Vitiligo-like depigmentation in patients with stage III-IV melanoma receiving immunotherapy and its association with survival: a systematic review and meta-analysis. J Clin Oncol. 2015;33(7):773-781. doi:10.1200/JCO.2014.57.4756

24. Abu-Sbeih H, Ali FS, Qiao W, et al. Immune checkpoint inhibitor-induced colitis as a predictor of survival in metastatic melanoma. Cancer Immunol Immunother. 2019;68(4):553-561. doi:10.1007/s00262-019-02303-1

25. Robert C, Hwu WJ, Hamid O, et al. Long-term safety of pembrolizumab monotherapy and relationship with clinical outcome: a landmark analysis in patients with advanced melanoma. Eur J Cancer. 2021;144:182-191. doi:10.1016/j.ejca.2020.11.010 
26. Eggermont AMM, Kicinski M, Blank CU, et al. Association between immune-related adverse events and recurrence-free survival among patients with stage III melanoma randomized to receive pembrolizumab or placebo: a secondary analysis of a randomized clinical trial. JAMA Oncol. 2020;6(4):519-527. doi:10.1001/jamaoncol.2019.5570

27. Eggermont AMM, Blank CU, Mandala M, et al. Adjuvant pembrolizumab versus placebo in resected stage III melanoma. $N$ Engl J Med. 2018;378:1789. doi:10.1056/NEJMoa1802357

28. Weber J, Mandala M, Del, Vecchio M, et al. Adjuvant nivolumab versus ipilimumab in resected stage III or IV melanoma. $N$ Engl J Med. 2017;377(19):1824-1835. doi:10.1056/NEJMoa1709030

29. Zimmer L, Livingstone E, Hassel JC, et al. Adjuvant nivolumab plus ipilimumab or nivolumab monotherapy versus placebo in patients with resected stage IV melanoma with no evidence of disease (IMMUNED): a randomized, double-blind, placebo-controlled, phase 2 trial. Lancet. 2020;395:1558. doi:10.1016/S0140-6736(20)30417-7

30. Kehl KL, Yang S, Awad MM, et al. Pre-existing autoimmune disease and the risk of immune-related adverse events among patients receiving checkpoint inhibitors for cancer. Cancer Immunol Immunother. 2019;68:817-926. doi:10.1007/s00262-019-02321-z

31. Larkin J, Chiarion-Sileni V, Gonzalez R, et al. Combined nivolumab and ipilimumab or monotherapy in untreated melanoma. $N$ Engl J Med. 2015;373:23-34. doi:10.1056/NEJMoa1504030

32. Bender DA, Heilbroner SP, Wang TJC, et al. Increased rates of immunosuppressive treatment and hospitalization after checkpoint inhibitor therapy in cancer patients with autoimmune disease. $J$ Immunother Cancer. 2020;8:e01627. doi:10.1136/jitc-2020-001627

33. Cortellini A, Buti S, Santini D, et al. Clinical outcomes of patients with advanced cancer and pre-existing autoimmune diseases treated with anti-programmed death-1 immunotherapy: a real-world transverse study. Oncologist. 2019;24:e327-e337. doi:10.1634/theoncologist.2018-0618

34. Abdel-Wahab N, Shah M, Lopez-Olivo MA, Suarez-Almazor ME. Use of checkpoint inhibitors in the treatment of patients with cancer and preexisting autoimmune disease: a systematic review. Ann Int Med. 2018;168(2):121-131. doi:10.7326/M17-2073

35. Leonardi GC, Gainor JF, Alltan M, et al. Safety of programmed death-1 pathway inhibitors among patients with non-small-cell lung cancer and preexisting autoimmune disorders. $J$ Clin Oncol. 2018;36:1905-1912.

36. Johnson DB, Sullivan RJ, Ott PA, et al. Ipilimumab therapy in patients with advanced melanoma and preexisting autoimmune disorders. JAMA Oncol. 2016;2(2):234-240. doi:10.1001/ jamaoncol.2015.4368

37. Kahler KC, Eigentler TK, Gesierich A, et al. Ipilimumab in metastatic melanoma patients with pre-existing autoimmune disorders. Cancer Immunol Immunother. 2018;67(5):825-834. doi:10.1007/ s00262-018-2134-z

38. Menzies AM, Johnson DB, Ramanujam S, et al. Anti-PD-1 therapy in patients with advanced melanoma and preexisting autoimmune disorders or major toxicity with ipilimumab. Ann Oncol. 2017;28 (2):368-376. doi:10.1093/annonc/mdw443

39. Bostwick AD, Salama AK, Hanks BA. Rapid complete response of metastatic melanoma in a patient undergoing ipilimumab immunotherapy in the setting of active ulcerative colitis. $J$ Immunother Cancer. 2015;3(3):19. doi:10.1186/s40425-015-0064-2

40. Grover S, Ruan AB, Srivoleti P, et al. Safety of immune checkpoint inhibitors in patients with pre-existing inflammatory bowel disease and microscopic colitis. JOP. 2020;16(9):e933-e941.
41. Abu-Sbeih H, Faleck DM, Riccitui B, et al. Immune checkpoint inhibitor therapy in patients with preexisting inflammatory bowel disease. J Clin Oncol. 2020;38(6):576-583. doi:10.1200/JCO.19.01674

42. Uemura M, Trinh VA, Haymaker C, et al. Selective inhibition of autoimmune exacerbation while preserving the anti-tumor clinical benefit using IL-6 blockade in a patient with advanced melanoma and Crohn's disease: a case report. J Hematol Oncol. 2016;9:81. doi:10.1186/s13045-016-0309-7

43. Frohne CC, Llano EM, Perkovic A, Cohen RD, Luke JL. Complete response of metastatic melanoma in a patient with Crohn's disease simultaneously receiving anti-alph4beta7 and anti-PD1 antibodies. $J$ Immunother Cancer. 2019;7(1):1. doi:10.1186/s40425-018-0484-x

44. Mellemkjaer L, Linet MS, Frisch M, et al. Rheumatoid arthritis and cancer risk. Eur J Cancer. 1996;32A(10):1753-1757. doi:10.1016/ 0959-8049(96)00210-9

45. Chen Y-J, Chang Y-T, Wang C-B, et al. The risk of cancer in patients with rheumatoid arthritis. Arthritis Rheum. 2011;63(2):352-358. doi:10.1002/art.30134

46. Klinaki E, Katsoulis M, La Vecchia C, et al. Rheumatoid arthritis and cancer risk results from the Greek European prospective investigation into cancer and nutrition cohort. Eur J Cancer Prev. 2018;27 (5):502-506. doi:10.1097/CEJ.0000000000000349

47. Leipe J, Christ LA, Arnoldi AP, et al. Characteristics and treatment of new-onset arthritis after checkpoint inhibitor therapy. RMD Open. 2018;4:e00714.

48. Cappelli LC, Brahmer JR, Forde PM, et al. Clinical presentation of immune checkpoint inhibitor-induced inflammatory arthritis differs by immunotherapy regimen. Semin Arthritis Rheum. 2018;48 (3):553-557. doi:10.1016/j.semarthrit.2018.02.011

49. Braaten TJ, Brahmer JR, Forde PM, et al. Immune checkpoint inhibitor-induced inflammatory arthritis persists after immunotherapy cessation. Ann Rheum Dis. 2020;79(3):332-338. doi:10.1136/ annrheumdis-2019-216109

50. Efuni E, Cytryn S, Boland P, et al. Risk of toxicity after initiating immune checkpoint inhibitor treatment in patients with rheumatoid arthritis. J Clin Rheum. 2020.

51. Lee B, Wong A, Kee D, et al. The use of ipilimumab in patients with rheumatoid arthritis and metastatic melanoma. Ann Oncol. 2016;27:1174-1177. doi:10.1093/annonc/mdw056

52. Kyi C, Carvajal RD, Wolchock JD, Postow MA. Ipilimumab in patients with melanoma and autoimmune disease. $J$ Immunother Cancer. 2014;2:35. doi:10.1186/s40425-014-0035-Z

53. Garcia CR, Jayswal R, Adams V, Anthony LB, Villano JL. Multiple sclerosis outcomes after cancer immunotherapy. Clin Transl Oncol. 2019;21:1336-1342. doi:10.1007/s12094-019-02060-8

54. Phadke SD, Ghabour R, Swick BL, Swenson A, Milhem M, Zakharia Y. Pembrolizumab therapy triggering an exacerbation of preexisting autoimmune disease: a report of 2 patient cases. $J$ Invest Med. 2016;4:1-15.

55. Lau KHV, Kumar A, Yang IH, et al. Exacerbation of myasthenia gravis in a patient with melanoma treated with pembrolizumab. Muscle Nerve. 2016;54:157-161. doi:10.1002/mus.25141

56. Hannen J, Ernstoff MS, Wang Y, et al. Autoimmune diseases and immune-checkpoint inhibitors for cancer therapy: review of the literature and personalized risk-based prevention strategy. Ann Oncol. 2020;31(6):724-744. doi:10.1016/j.annonc.2020.03.285

57. Jia XH, Geng LY, Jiang PP, et al. The biomarkers related to immune related adverse events caused by immune checkpoint inhibitors. $J$ Exp Clin Cancer Res. 2020;39(1):2. doi:10.1186/s13046-020-01749-x 


\section{Publish your work in this journal}

Cancer Management and Research is an international, peer-reviewed open access journal focusing on cancer research and the optimal use of preventative and integrated treatment interventions to achieve improved

The manuscript management system is completely online and includes a very quick and fair peer-review system, which is all easy to use. Visit http://www.dovepress.com/testimonials.php to read real quotes outcomes, enhanced survival and quality of life for the cancer patient.

from published authors.

Submit your manuscript here: https://www.dovepress.com/cancer-management-and-research-journa 